Kategorien, die zwischen den Beobachtern nur mehr oder weniger übereinstimmen.

Sieht man von diesen definitorischen Fallstricken ab (die nur wegen ihrer grundsätzlichen Bedeutung hier so ausführlich behandelt wurden), hat Engesser eine wichtige empirische Studie vorgelegt, die durch ihre Breite (112 deutsch- und englischsprachige Webangebote, 30 Qualitätskriterien), durch den kombinierten Einsatz von Befragung und Inhaltsanalyse sowie der Sorgfalt bei der Herleitung von Qualitätskriterien, der Auswertung und beim Vergleich der Befunde mit anderen Studien ein großer Gewinn ist. Mit Hilfe einer Clusteranalyse konnte er drei Typen von partizipativ-journalistischen Websites ermitteln: kommerzielle Spielwiesen, lokale Medienspiegel und exklusive Themenseiten. Die Angebote schneiden gut ab bei den Kriterien Argumentativität, Authentizität, Rechtmäßigkeit und Richtigkeit; Schwächen besitzen sie u. a. bei den Kriterien Gleichheit, Objektivität, Publizität, Relevanz und Transparenz. Den partizipativen Journalismus kennzeichnen der Bezug auf sich selbst und die direkte Lebenswelt, ein Hang zum Positivismus und zur Sympathiewerbung sowie eine Vernachlässigung der Trennung von Nachricht und Meinung. Einen positiven Einfluss auf die Gesamtqualität hat besonders die Zahl der Wettbewerber, während sich die Existenz eines Muttermediums eher negativ auswirkt.

Christoph Neuberger

\section{Uwe Krüger}

\section{Meinungsmacht}

Der Einfluss von Eliten auf Leitmedien und

Alpha-Journalisten - eine kritische

Netzwerkanalyse

Köln: Halem, 2013. - 375 Seiten

(Reihe des Instituts für Praktische

Journalismus- und Kommunikationsforschung [IPJ]; 9)

ISBN 978-3-86962-070-1

(Zugl.: Leipzig, Univ., Diss., 2011)

In Meinungsmacht untersucht Uwe Krüger das Zusammenspiel von journalistischen, politischen und wirtschaftlichen Eliten vor dem Hintergrund eines Diskurses über die Grundausrichtung der deutschen Sicherheitspolitik. Forschungsleitend ist die Frage, welcher Zusammenhang zwischen einer Elitenorientierung deutscher Leitmedien einerseits und ihrer Berichterstattung über das Themenfeld „Sicherheit, Verteidigung und Auslandseinsätze der
Bundeswehr" (S. 29) konstatiert werden kann. Der Autor selbst verortet seine Arbeit als „Mittelweg" zwischen einer liberal-pluralistischen und einer marxistischen Tradition. Sie soll mit einer „kritischen Grundhaltung“ das Netzwerk führender Journalisten zu Politik- und Wirtschaftseliten aufarbeiten und dabei die Frage beantworten, wie sich die Medien dieser Journalisten durch eine inhaltliche Elitenorientierung auszeichnen (S. 28). Beeinflusst also die Meinung der Eliten die Auswahl der Nachrichten und die Stoßrichtung der Kommentare?

Für seine empirische Untersuchung kombiniert der Autor die Soziale Netzwerkanalyse mit einer Inhaltsanalyse. Die für die Netzwerkanalyse notwendige Identifikation von Journalistennetzwerken und die damit $\mathrm{zu}$ beschreibenden personellen und institutionellen Kontakte zu gesellschaftlichen Eliten bildeten eine wichtige Basis, für die Krüger zunächst eine umfangreiche Dokumentenanalyse und Recherche durchgeführt hat. Für die Erfassung der Grundgesamtheit wählte er die Positionseliten deutscher Leitmedien aus. Letztere selektierte er auf Basis zweier Journalistenbefragungen (u. a. JouriD 2005). Insgesamt 219 Führungspositionen, die von den Herausgebern bis zu den Leitungen der Berliner Büros reichten, flossen in die Analyse ein (S. 111). In 82 Organisationen verfügten insgesamt 64 der journalistischen Positionen über ein Kontaktpotenzial mit politischen und wirtschaftlichen Eliten (Kap. 5). Vier außenpolitisch tonangebende Journalisten (K.-D. Frankenberger, J. Joffe, S. Kornelius, M. Stürmer), die innerhalb dieses Netzwerks auch allgemein zu den am stärksten eingebundenen Akteuren zählen, fielen durch sich mehrfach überlappende Kontakte bzw. Organisationsbeziehungen (z. B. Münchener Sicherheitskonferenz) auf.

Inwieweit die auf diese Weise aufgedeckte Nähe zum „transatlantischen Elitenmilieu“ (S. 151) auch inhaltlichen Niederschlag findet, analysiert Krüger anhand der Kommentare und Leitartikel der genannten vier Journalisten in den Jahren 2002-2010 zur deutschen oder europäischen Außen- und Sicherheitspolitik (Kap. 6). Die Analyse, die mit umfangreichen Zitationen aus den Beiträgen einhergeht, wird ergänzt um eine spezifischere Inhaltsanalyse der Berichterstattung zur Münchener Sicherheitskonferenz (Kap. 7). In dieser Teilstudie wird die Berichterstattung von SZ, FAZ, Welt, bei denen drei der vier oben genannten Journalisten mit Elite-Bezug arbeiten, mit der Berichterstattung von FR und TAZ in den Jahren 2007 bis 2010 verglichen. 
Ziel der Analyse ist offenkundig der Versuch, den festgestellten Elitendiskurs mit alternativen Perspektiven $\mathrm{zu}$ kontrastieren und wieder $\mathrm{zu}$ „entindividualisieren“ (S. 257). Nicht nur die Medienauswahl spiegelt dies wieder, sondern auch der in dieser Analyse verschobene Fokus, der neben der Berichterstattung mit Konferenzbezug auch alle Beiträge aufnimmt, die die Gegenveranstaltungen und Proteste gegen die Sicherheitskonferenz thematisieren.

Das Vorgehen ist insgesamt nachvollziehbar beschrieben. Die Diskussion der vielfältigen Ergebnisse, die eine einseitige Berichterstattung der vier im Detail untersuchten Journalisten nahelegen, wird von verschiedenen Seiten geführt. Der Autor stellt insbesondere eine normative Dimension und Schlussfolgerung in den Vordergrund: „Qualitätsjournalismus [sollte] den Elitendiskurs transzendieren" (S. 263), womit gemeint ist, dass Journalisten die darin enthaltenen Argumente kritisch würdigen und um nicht repräsentierte Meinungen ergänzen sollten. In der Konsequenz fordert Uwe Krüger auch, einen „Sicherheitsabstand“ zu definieren (S. 264). Dieser sollte Aufgaben und Funktionen von Journalisten in Beiräten, Kuratorien und vor allem in Politikplanungskörperschaften ausschließen, sofern Berührungspunkte zum Berichterstattungsfeld bestehen. Neben dieser diskussionswürdigen Empfehlung stellt sich allerdings die Frage, ob die Perspektive der vier kritisch betrachteten Journalisten stellvertretend für die in ihren Medien repräsentierten Meinungen zum Sicherheitsbegriff steht. Diese Frage beantwortet die Dissertation nicht eindeutig, da die dritte Teilstudie allein auf die Sicherheitskonferenz in München zugeschnitten ist und nicht breiter das ursprüngliche Feld der Außen- und Sicherheitspolitik mit Deutschlandbezug thematisiert. Ob also Gegenmeinungen im Sinne eines Binnenpluralismus in SZ, FAZ, Welt und Zeit zum „Kommentariat" oder der „Propaganda“ (S. 257) der vier Journalisten veröffentlicht wurden, wird letztlich nicht klar erhellt.

Die Theorien, auf denen Krüger seine Arbeit aufbaut, stammen größtenteils aus amerikanischer Feder. Diese kritische Weitung des Blicks macht das Buch spannend. Erläutert werden u. a. die Indexing-Hypothese (Bennett), das Propagandamodell (Herman/Chomsky), die „Guard Dog-Perspective“ (Donohue et al.) sowie das „Protestparadigma“ (McLeod/Hertog). Die genannten Ansätze liefern Bausteine zur Erklärung von Elitenkonformität in den Medien. Lesenswert sind auch die Dokumentation der Hintergrundkreise deutscher Journalisten (S. 34) sowie die Darstellung der Eli- tenkontakte im zweiten Kapitel. Die theoretische Basis der Arbeit ist auf die genannten Ansätze fokussiert. Weitere Anknüpfungspunkte hätten aus Sicht des Rezensenten allerdings auch die anderenorts vorgelegten Befunde $\mathrm{zu}$ medienöffentlichen Diskursen mit der Betonung einer bipolaren Konfliktstruktur (vgl. Weßler 1999) bieten können sowie die durchaus differenzierten Ergebnisse zur Sichtbarkeit zivilgesellschaftlicher Akteure in deutschen $\mathrm{Me}-$ dien.

Mit Blick auf den hier untersuchten (Elite-)Diskurs liefert Uwe Krügers Arbeit insgesamt einen kritischen Ankerpunkt, der für Journalisten, Studierende und Wissenschaftler gleichermaßen empfehlenswert ist. Weitere Forschung, die der Ausgewogenheitsnorm in solchen, in Leitmedien geführten Debatten empirisch auf den Zahn fühlt, ist wünschenswert.

Christian Nuernbergk

\section{Margreth Lünenborg / Tanja Maier \\ Gender Media Studies \\ Eine Einführung}

Konstanz: UVK, 2013. - 224 S.

ISBN 978-3-8252-3872-8

Zusatzmaterialien unter: http://

www.polsoz.fu-berlin.de/kommwiss/

arbeitstellen/journalistik/media/2013-03-06-

Zusatzmaterial_Gender_media_Studies.pdf? 1367707886

Ein Lehrbuch zur kommunikationswissenschaftlichen Geschlechterforschung ist im deutschsprachigen Raum längst überfällig. Margreth Lünenborg und Tanja Maier haben es nun vorgelegt. „Gender Media Studies - eine Einführung" besteht aus drei Teilen sowie einem ausführlichen Literaturverzeichnis, das Kernliteratur auf aktuellem Stand bereitstellt. Im deutschsprachigen Raum für Lehrbücher noch keineswegs selbstverständlich, werden wichtige Begriffe in Kästchen hervorgehoben, am Ende jedes Kapitels finden sich Fragen und Literaturempfehlungen und im Teil III zusätzlich noch Übungsaufgaben.

Im Teil I stehen zunächst „Theorien und Konzepte“ im Mittelpunkt. Hier finden sich Informationen zur Geschichte des interdisziplinären Forschungsfelds, Definitionen zentraler Begriffe und schließlich eine hilfreiche Erweiterung der bis dato gängigen Einteilung der drei Ansätze der Geschlechterforschung: Neben Gleichbeits- und Differenzansatz werden der interaktionistische Konstruktivismus und 